\title{
Sommes friables de fonctions multiplicatives aléatoires ${ }^{1}$
}

\author{
Joseph Basquin
}

\begin{abstract}
We consider a sequence $\{f(p)\}_{p}$ prime of independent random variables taking values \pm 1 with probability $1 / 2$, and extend $f$ to a multiplicative arithmetic function defined on the squarefree integers. We investigate upper bounds for $\Psi_{f}(x, y)$, the summatory function of $f$ on $y$-friable integers $\leqslant x$.

We obtain estimations of the type $\Psi_{f}(x, y) \ll \Psi(x, y)^{1 / 2+\varepsilon}$, more precise formulas being given in suitable regions for $x, y$.

In the special case $y=x$, this leads to the estimate $M_{f}(x)=$ $\sum_{n \leqslant x} f(n) \ll \sqrt{x}(\log \log x)^{2+\varepsilon}$, which improves on previous bounds.
\end{abstract}

\section{Sommaire}

1 Introduction et description des résultats $\ldots \ldots \ldots \ldots \ldots \ldots \ldots \ldots \ldots \ldots \ldots$

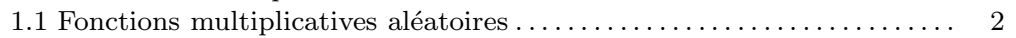

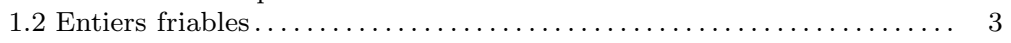

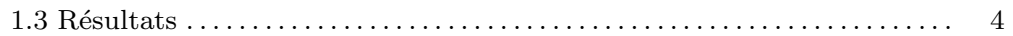

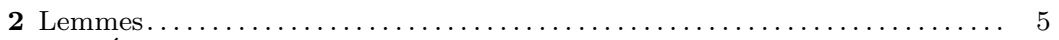

2.1 Évaluation d'une somme de fonction multiplicative $\ldots \ldots \ldots \ldots \ldots \ldots .5$

2.2 Espérance de fonctions multiplicatives aléatoires $\ldots \ldots \ldots \ldots \ldots \ldots \ldots$

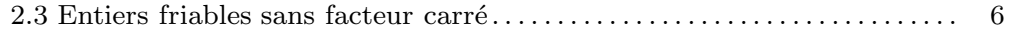

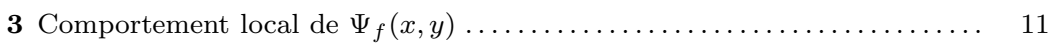

3.1 Martingale $y \mapsto \Psi_{f}(x, y)$ et inégalité maximale $\ldots \ldots \ldots \ldots \ldots \ldots \ldots \ldots 11$

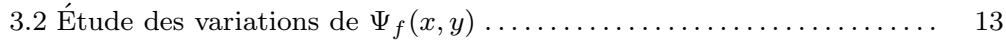

3.3 Moyenne de $\Psi_{f}(x, y)$ autour de points-tests $\ldots \ldots \ldots \ldots \ldots \ldots \ldots \ldots 17$

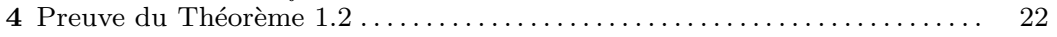

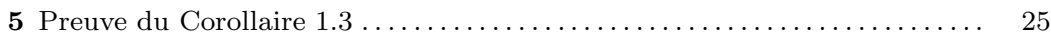

2010 Mathematics Subject Classification. Primary : 11N25, 11N37. Secondary : 60G42. Key words and phrases : multiplicative functions, random multiplicative functions, friable integers, martingales, Doob's inequality.

1 Version au 18/09/2011. 
JOSEPH BASQUIN

\section{Introduction et description des résultats}

\subsection{Fonctions multiplicatives aléatoires}

L'étude des sommes de fonctions multiplicatives est un domaine central de la théorie analytique des nombres. Ces fonctions possèdent, dans la plupart des cas intéressants, un comportement statistique complexe.

D'où l'idée, mise en pratique par plusieurs auteurs, de considérer des fonctions multiplicatives aléatoires, dont les variations, que l'on peut appréhender par les outils de la théorie des probabilités, fournissent un modèle pertinent de la situation arithmétique. Selon les hypothèses effectuées sur les variables aléatoires en cause, de tels modèles peuvent être adaptés aux diverses situations concrètes rencontrées en théorie des nombres.

La voie privilégiée par Wintner dans [14] consiste à considérer, pour chaque nombre premier $p$, une variable aléatoire de Bernoulli $f(p)$, prenant les valeurs 1 et -1 avec probabilité $1 / 2$, et à étendre par multiplicativité cette fonction $f$ à l'ensemble des entiers sans facteur carré. Ceci donne lieu à la définition suivante.

Définition 1.1. Notant $\mathcal{P}$ l'ensemble des nombres premiers, soient $\{f(p)\}_{p \in \mathcal{P}}$ une suite de variables aléatoires de Bernoulli indépendantes prenant les valeurs +1 et -1 avec probabilité $1 / 2$, et $f$ la fonction multiplicative définie pour $n \in \mathbb{N}^{*}$ par

$$
f(n):=\mu(n)^{2} \prod_{p \mid n} f(p) .
$$

Sous ces hypothèses, nous dirons que $f$ est une fonction multiplicative aléatoire au sens de Wintner.

Posons

$$
M_{f}(x):=\sum_{n \leqslant x} f(n) \quad(x \geqslant 1) .
$$

Une mesure de l'indépendance statistique des facteurs premiers des entiers est obtenue en comparant la fonction sommatoire de telles fonctions multiplicatives aléatoires à celle d'un modèle probabiliste, comme par exemple celui d'une somme de variables aléatoires centrées indépendantes relevant du théorème central limite.

Citons un premier résultat de Wintner [14] allant dans cette direction. Nous utilisons dorénavant la mention ps pour qualifier une assertion aléatoire valide presque sûrement.

Théorème A. Soit $f$ une fonction multiplicative aléatoire au sens de Wintner. Pour tout $\varepsilon>0$, nous avons

$$
M_{f}(x) \ll x^{1 / 2+\varepsilon} \quad(x \geqslant 1) \quad \text { ps. }
$$


L'enjeu essentiel du problème est de comparer le cas étudié ici au cas sans contrainte arithmétique, c'est-à-dire $f(n)= \pm 1$ avec probabilité $1 / 2$, pour lequel la loi du logarithme itéré fournit l'estimation ${ }^{(1)}$

$$
M_{f}(x) \ll \sqrt{x \log _{2} x},
$$

et ainsi d'élucider l'influence de la condition arithmétique de multiplicativité.

Dans un travail non publié, Erdős obtient l'existence d'une constante $c_{1}>0$ pour laquelle on a la majoration

$$
M_{f}(x) \ll \sqrt{x}(\log x)^{c_{1}} \quad \text { ps. }
$$

Halász [5] précise cette majoration et établit le résultat suivant.

Théorème B. Soit $f$ une fonction multiplicative aléatoire au sens de Wintner. Il existe une constante $c_{2}>0$ telle que l'on ait

$$
M_{f}(x) \ll \sqrt{x} \mathrm{e}^{c_{2} \sqrt{\left(\log _{2} x\right) \log _{3} x}} \quad(x \geqslant 16) \quad \text { ps. }
$$

Dans une version préliminaire [9] (améliorée depuis) d'un récent travail, Lau, Tenenbaum et $\mathrm{Wu}$ précisent encore cette majoration et obtiennent, pour une fonction multiplicative aléatoire au sens de Wintner $f$,

$$
M_{f}(x) \ll \sqrt{x}\left(\log _{2} x\right)^{5 / 2+\varepsilon} \quad \text { ps. }
$$

\subsection{Entiers friables}

Nous utilisons la notation $P^{+}(n)$ (resp. $\left.P^{-}(n)\right)$ pour le plus grand (resp. petit) facteur premier d'un entier $n$ avec la convention $P^{+}(1):=1$ (resp. $\left.P^{-}(1):=\infty\right)$. Un entier dont le plus grand facteur premier ne dépasse pas $y$ est dit $y$-friable. Nous désignons par $S(x, y)$ l'ensemble des entiers $y$-friables inférieurs ou égaux à $x$ et par $\Psi(x, y)$ son cardinal. Pour une fonction arithmétique $f$, nous posons de plus

$$
\Psi_{f}(x, y):=\sum_{n \in S(x, y)} f(n) .
$$

Nous notons

$$
\Psi^{*}(x, y):=\Psi_{\mu^{2}}(x, y)
$$

pour le nombre des entiers $y$-friables, sans facteur carré et n'excédant pas $x$.

L'étude de la friabilité et de son interaction avec les critères usuels de description utilisés en théorie probabiliste des nombres constitue une branche essentielle de

1. Ici et dans la suite, nous désignons par $\log _{k}$ la k-ième itérée de la fonction logarithme. 
l'arithmétique. Ce sujet fait l'objet d'une importante littérature depuis les années 1950, en particulier ces vingt dernières années.

\subsection{Résultats}

Nous nous proposons ici d'étudier les sommes friables $\Psi_{f}(x, y)$ d'une fonction multiplicative aléatoire au sens de Wintner $f$.

Un raisonnement statistique laissant augurer que les termes de la somme se comportent comme des variables aléatoires indépendantes, il est raisonnable d'espérer que la somme se comporte comme la racine carrée du nombre de termes, éventuellement multipliée par un facteur à faible croissance en $x$ et $y$.

Posons $\vartheta(y):=\sum_{p \leqslant y} \log p$. Nous pouvons à présent énoncer notre résultat principal.

Théorème 1.2. Soit $f$ une fonction multiplicative aléatoire au sens de Wintner. Pour tout $\varepsilon>0$, il existe des constantes positives $c_{3}, c_{4}, c_{5}, c_{6}$, telles que nous ayons presque sûrement,

$$
\Psi_{f}(x, y) \begin{cases}\ll \sqrt{x}\left(\log _{2} x\right)^{2+\varepsilon} & \text { si } x^{c_{3}\left(\log _{3} x\right) / \log _{2} x} \leqslant y \leqslant x \\ & \text { et } x \geqslant 16, \\ \ll \sqrt{\Psi^{*}(x, y)} \mathrm{e}^{c_{4} u}(\log x)^{c_{5}} & \text { si } c_{6} \log x \leqslant y<x^{c_{3}\left(\log _{3} x\right) / \log _{2} x}, \\ & \text { et } x \geqslant 16 .\end{cases}
$$

Remarques. (i) Dans le cas particulier $y=x$, il est à noter que le théorème précédent fournit une amélioration du résultat $(1 \cdot 1)$ :

$$
M_{f}(x) \ll \sqrt{x}\left(\log _{2} x\right)^{2+\varepsilon} \quad \text { ps. }
$$

(ii) Lorsque $\vartheta(y) \leqslant \log x$ et $x \geqslant 2$, nous avons

$$
\Psi_{f}(x, y)= \begin{cases}0 & \text { avec probabilité } 1-2^{-\pi(y)}, \\ 2^{\pi(y)} & \text { avec probabilité } 2^{-\pi(y)}\end{cases}
$$

En effet, dans ce domaine, on a

$$
\Psi_{f}(x, y)=\sum_{P^{+}(n) \leqslant y} f(n)=\prod_{p \leqslant y}(1+f(p))=0
$$

si et seulement s'il existe $p \leqslant y$ tel que $f(p)=-1$, ce qui intervient avec probabilité $1-2^{-\pi(y)}$.

Le Théorème 1.2 implique le résultat suivant.

Corollaire 1.3. Soient $f$ une fonction multiplicative au sens de Wintner et $\varepsilon>0$. Il existe une constante une constante positive $C_{\varepsilon}$ telle que, pour $x, y \geqslant 2$ et $y \geqslant C_{\varepsilon} \log x$, nous ayons

$$
\Psi_{f}(x, y) \ll \Psi^{*}(x, y)^{1 / 2+\varepsilon} .
$$




\section{Lemmes}

\section{1. Évaluation d'une somme de fonction multiplicative}

Nous désignons par $\sum_{n}^{y, z}$ une sommation dont l'indice entier $n$ est soumis à la condition $p \mid n \Rightarrow y<p \leqslant z$.

Pour $y \geqslant 2$, nous posons de plus $L(y):=\exp \left\{(\log y)^{3 / 5} /\left(\log _{2} y\right)^{1 / 5}\right\}$.

La majoration suivante, obtenue par Lau, Tenenbaum et $\mathrm{Wu}([9])$ pour certaines sommes de fonctions multiplicatives, prolonge le lemme 3 de [5].

Lemme 2.1. Soient $\gamma, \delta>0, y \geqslant 2, \kappa \geqslant 1$ et $a, b \in \mathbb{N}^{*}$ tels que $b>a$. Il existe des constantes $c_{7}, c_{8}, c_{9}>0$ telles que nous ayons, uniformément pour $\gamma \geqslant c_{8} / L(y)^{c_{9}}$ et $y \geqslant\{b /(b-a)\}^{1+\delta}$,

$$
\sum_{a<r \leqslant b}^{y, y^{1+\gamma}} \mu(r)^{2} \kappa^{\omega(r)} \leqslant c_{7} \frac{b-a}{\log y} \kappa \mathrm{e}^{2 \gamma \kappa} .
$$

Démonstration. En suivant la méthode employée par Halász dans [5] (lemme 3 (ii)), il vient

$$
A:=\sum_{a<r \leqslant b}^{y, y^{1+\gamma}} \mu(r)^{2} \kappa^{\omega(r)} \leqslant \frac{1}{\log a} \sum_{m \leqslant b / y}^{y, y^{1+\gamma}} \mu(m)^{2} \kappa^{\omega(m)+1} \sum_{a / m<p \leqslant b / m} \log p .
$$

Notons que l'on peut supposer $b>y$, car dans le cas contraire, la somme à estimer est nulle. Observons également que les hypothèses $y \geqslant\{b /(b-a)\}^{1+\delta}$ et $m \leqslant b / y$ impliquent

$$
\frac{b-a}{m} \geqslant \frac{b}{m y^{1 /(1+\delta)}} \geqslant\left(\frac{b}{m}\right)^{1-1 /(1+\delta)}>1 .
$$

Le théorème de Brun-Titchmarsh (voir [10], théorème I.4.16) nous permet alors, pour $m \leqslant b / y$, de majorer la somme en $p$ de la façon suivante

$$
\sum_{a / m<p \leqslant b / m} \log p \leqslant \log (b / m) \sum_{a / m<p \leqslant b / m} 1 \leqslant c_{10} \frac{(b-a) \log (b / m)}{m \log ((b-a) / m)} .
$$

La relation $(2 \cdot 1)$ implique par ailleurs que

$$
\begin{aligned}
A & \leqslant c_{10} \frac{(b-a) \kappa \log (b / m)}{(\log a) \log ((b-a) / m)} \sum_{m \leqslant b / y}^{y, y^{1+\gamma}} \frac{\mu(m)^{2} \kappa^{\omega(m)}}{m} \\
& \leqslant c_{10} \frac{(b-a) \kappa}{\log a} \prod_{y<p \leqslant y^{1+\gamma}}\left(1+\frac{\kappa}{p}\right) .
\end{aligned}
$$


On conclut en faisant usage de l'estimation suivante, découlant par exemple du lemme 3.6 de [3],

$$
\sum_{y<p \leqslant y^{1+\gamma}} \frac{1}{p}=\log (1+\gamma)+O\left(\frac{1}{L(y)^{c_{9}}}\right) \leqslant 2 \gamma,
$$

dès lors que $\gamma \geqslant c_{8} / L(y)^{c_{9}}$.

\subsection{Espérance de fonctions multiplicatives aléatoires}

Le lemme suivant est dû Bonami [1]. Halász ([5], lemme 2) en a donné une nouvelle démonstration. La formulation donnée ici est adaptée au cas des fonctions multiplicatives aléatoires.

Lemme 2.2. Soient $f$ une fonction multiplicative aléatoire au sens de Wintner, $\ell \geqslant 2$ un entier pair, et $\left\{a_{n}\right\}_{n \geqslant 1}$ une suite de nombres complexes. Nous avons

$$
\left|\mathbb{E}\left\{\left(\sum_{n \geqslant 1} a_{n} f(n)\right)^{\ell}\right\}\right| \leqslant\left(\sum_{n \geqslant 1} \mu(n)^{2}\left|a_{n}\right|^{2}(\ell-1)^{\Omega(n)}\right)^{\ell / 2} .
$$

Cette majoration s'avère cruciale pour évaluer les moments d'ordre pair de fonctions multiplicatives aléatoires.

Remarque. Pour $\ell=2$ et $\left\{a_{n}\right\}_{n} \geqslant 1$ égale à la fonction indicatrice des entiers $y$ friables inférieurs à $x$, la majoration fournie par le lemme précédent est en fait une égalité. Nous avons en effet

$$
\mathbb{E}\left(\Psi_{f}(x, y)^{2}\right)=\Psi^{*}(x, y) \quad(x, y \geqslant 2) .
$$

Cela résulte du développement du carré sous la forme

$$
\mathbb{E}\left(\Psi_{f}(x, y)^{2}\right)=\mathbb{E}\left\{\sum_{m, n \in S(x, y)} f(m) f(n)\right\}=\sum_{m, n \in S(x, y)} \mathbb{E} f(m) f(n) .
$$

Il suffit ensuite d'observer que, si $m$ et $n$ sont sans facteur carré et $m \neq n$, il existe un nombre premier $p$ tel que $p \| m n .^{(2)}$ Il en découle

$$
\mathbb{E}(f(m) f(n))=\mathbb{E}(f(p)) \mathbb{E}(f(m n / p))=0 .
$$

\subsection{Entiers friables sans facteur carré}

Le résultat suivant, obtenu par La Bretèche et Tenenbaum [2], fournit une description de la répartition des entiers friables sans facteur carré dans les petits intervalles.

2. Ici $p^{\nu} \| a$ signifie $: p^{\nu} \mid a$ et $p^{\nu+1} \nmid a$. 
Lemme 2.3. Soit $\kappa \geqslant 1$. Nous avons, uniformément sous les conditions $x \geqslant y \geqslant 2$ et $\max \left(1, x y^{-\kappa}\right) \leqslant z \leqslant x$,

$$
\Psi^{*}(x+z, y)-\Psi^{*}(x, y) \ll \frac{z}{x} \Psi^{*}(x, y) .
$$

Posons à présent $\varphi(s, y):=\sum_{p \leqslant y} \log \left(1+1 / p^{s}\right)(s \geqslant 0)$ et désignons par $\alpha=\alpha(x, y)$ l'unique solution positive de l'équation

$$
-\varphi^{\prime}(\alpha, y)=\sum_{p \leqslant y} \frac{\log p}{1+p^{\alpha}}=\log x
$$

Par ailleurs, nous désignons $\operatorname{par} \beta:=\beta(x, y)$ l'unique solution positive de l'équation

$$
\sum_{p \leqslant y} \frac{\log p}{p^{\beta}-1}=\log x .
$$

Pour $t>0, t \neq 1$, nous définissons $\xi(t)$ comme l'unique solution réelle non nulle de l'équation

$$
\mathrm{e}^{\xi}=1+t \xi
$$

et posons $\xi(1)=0$.

Rappelons que pour $\varepsilon>0, x \geqslant x_{0}(\varepsilon)$ et $(\log x)^{1+\varepsilon}<y \leqslant x$, nous avons les estimations (voir (7.8) de [7] et (2.17) de [2])

$$
\left.\begin{array}{l}
\alpha(x, y) \\
\beta(x, y)
\end{array}\right\}=1-\frac{\xi(u)}{\log y}+O\left(\frac{1}{L_{\varepsilon}(y)}+\frac{1}{u(\log y)^{2}}\right)
$$

où nous avons posé

$$
L_{\varepsilon}(y):=\exp \left\{(\log y)^{3 / 5-\varepsilon}\right\} .
$$

Le résultat suivant est un analogue du théorème 2.4 de [3], énoncé ici dans le cas des entiers friables sans facteur carré.

Lemme 2.4. Il existe des constantes absolues positives $b_{1}, b_{2}$, et une fonction $b=b(x, y ; d) \in\left[b_{1}, b_{2}\right]$ telles que, sous les conditions

$$
x \geqslant y \geqslant 2, \quad \vartheta(y)>2 \log x, \quad 1 \leqslant d \leqslant x,
$$

nous ayons uniformément

$$
\Psi^{*}\left(\frac{x}{d}, y\right)=\frac{\Psi^{*}(x, y)}{d^{\alpha}} \mathrm{e}^{-b t^{2} / u}\left\{1+O\left(\frac{t}{\sqrt{u}}+\frac{1}{u_{y}}\right)\right\},
$$

où $t:=(\log d) / \log y, u_{y}:=u+(\log y) / \log (u+2)$, et $\alpha=\alpha(x, y)$ est défini par $(2 \cdot 3)$. 
Démonstration. Notons que nous pouvons supposer $x$ et $y$ assez grands. En effet, lorsque $x$ est borné, le résultat est acquis sous réserve que les constantes implicites des termes d'erreurs soient choisies suffisamment grandes. Si $y$ est borné, la relation $\vartheta(y)>2 \log x$ implique que $x$ est borné à son tour, d'où le résultat. Nous supposons donc dans toute la suite que $x$ et $y$ sont suffisamment grands.

Plaçons-nous tout d'abord dans le domaine

$$
y>(\log x)^{3}, \quad x \geqslant x_{0} .
$$

Nous pouvons faire appel aux résultats de [3] concernant le comportement local de $\Psi(x, y)$. Au vu de l'estimation (2.4), nous avons $\beta \geqslant 3 / 5$, dès que $y$ est assez grand. Les quantités $\zeta(2 \beta, y)$ et $\prod_{p \leqslant y}\left(1+p^{-2 \beta}\right)$ étant bornées, le corollaire 2.6 de [3] nous permet alors d'écrire

$$
\Psi^{*}(x, y)=\frac{\Psi(x, y)}{\zeta(2 \beta, y)}\left\{1+O\left(\frac{1}{u_{y}}\right)\right\}
$$

où nous avons posé, pour $y \geqslant 2$,

$$
\zeta(s, y):=\sum_{P^{+}(n) \leqslant y} \frac{1}{n^{s}}=\prod_{p \leqslant y}\left(1-\frac{1}{p^{s}}\right)^{-1} .
$$

Nous avons d'une part

$$
\zeta(2 \beta, y)=\zeta(2 \beta)+O\left(1 / u_{y}\right)
$$

et d'autre part,

$$
\sum_{p \leqslant y} \frac{(\beta-\alpha)(\log p)^{2}}{p^{\beta}-1} \ll \sum_{p \leqslant y} \frac{\left(p^{\beta}-p^{\alpha}\right) \log p}{\left(p^{\alpha}+1\right)\left(p^{\beta}-1\right)}=\sum_{p \leqslant y} \frac{2 \log p}{\left(p^{\alpha}+1\right)\left(p^{\beta}-1\right)} \ll 1
$$

ce qui implique encore

$$
\beta-\alpha \ll 1 / u(\log y)^{2} .
$$

Le théorème 2.4 de [3] implique alors

$$
\Psi^{*}\left(\frac{x}{d}, y\right)=\frac{\Psi^{*}(x, y)}{d^{\alpha}} \exp \left\{\frac{-b t^{2}}{u}+(\alpha-\beta) \log d\right\}\left\{1+O\left(\frac{t}{u}+\frac{1}{u_{y}}\right)\right\}
$$

sous la condition $(2 \cdot 6)$, ce qui fournit bien la formule $(2 \cdot 5)$ dans ce domaine. De plus, on peut y remplacer $t / \sqrt{u}$ par $t / u$ dans le terme d'erreur.

Il reste à examiner le cas

$$
x \geqslant x_{0}, \quad 2 \log x<\vartheta(y) \leqslant(\log x)^{3},
$$


Nous pouvons nous limiter à prouver le résultat lorsque $t \leqslant u-1$, i.e. $d \leqslant x / y$. En effet, si nous supposons le résultat établi dans ce sous-domaine, nous avons, lorsque $x / y<d \leqslant x$,

$$
\begin{aligned}
\Psi^{*}\left(\frac{x}{d}, y\right) \asymp \frac{x}{d}=\frac{x}{d y} y & \ll \frac{x}{d y} \frac{\Psi^{*}(x, y)}{(x / y)^{\alpha}} \sqrt{u} \mathrm{e}^{-b(u-1)^{2} / u} \\
& \ll \frac{\Psi^{*}(x, y)}{d^{\alpha}} \mathrm{e}^{-b u / 2} \ll \frac{\Psi^{*}(x, y)}{d^{\alpha}} \mathrm{e}^{-b t^{2} / 2 u},
\end{aligned}
$$

car $\alpha<1$ d'après le lemme 2.8 de [2] sous la forme

$$
\alpha=\left\{1+O\left(\frac{1}{\log y}\right)\right\} \frac{\log (1+z)}{\log y},
$$

où $z:=z(x, y)>0$ est implicitement défini par la relation $\vartheta(y)=(2+z) \log y$.

Notons que dans le domaine considéré, nous avons $u \gg u_{y}$ et le théorème 2.1 de [2] fournit, pour $1 \leqslant d \leqslant x / y$,

$$
\Psi^{*}\left(\frac{x}{d}, y\right)=\mathrm{e}^{h(u-t)}\left\{1+O\left(\frac{t+1}{u}\right)\right\}
$$

où nous avons posé

$$
\begin{gathered}
h(v):=\alpha_{v} v \log y+\varphi(\alpha, y)+g\left(\alpha \sqrt{\sigma_{2}}\right), \quad \alpha_{v}:=\alpha\left(y^{v}, y\right) \quad(v \geqslant 1), \\
g(z):=\log \left\{\frac{\mathrm{e}^{z^{2} / 2}}{\sqrt{2 \pi}} \int_{z}^{\infty} \mathrm{e}^{-t^{2} / 2} \mathrm{~d} t\right\}, \quad \sigma_{2}:=\frac{\mathrm{d}^{2} \varphi}{\mathrm{d} s^{2}}(\alpha, y) .
\end{gathered}
$$

Il est établi en (2.35) de [2] que l'on a,

$$
h^{\prime}(v)=\alpha_{v} \log y+O\left(\frac{1}{\sqrt{v}+v \log (1+z)}+\frac{1}{v}\right) \quad(v \geqslant 1) .
$$

Il vient alors, pour $t \leqslant u-1$,

$$
h(u-t)=h(u)-\alpha_{u} t \log y-I+R,
$$

avec $R \ll \int_{u-t}^{u} \mathrm{~d} v / \sqrt{v} \ll t / \sqrt{u}$ et

$$
I:=(\log y) \int_{u-t}^{u}\left\{\alpha_{v}-\alpha_{u}\right\} \mathrm{d} v \asymp \int_{u-t}^{u} \int_{v}^{u} \frac{\mathrm{d} w \mathrm{~d} v}{w}=-(u-t) \log \left(\frac{u}{u-t}\right)+t,
$$

grâce à l'esimation $\alpha_{v}^{\prime} \asymp-1 /(v \log y)(v \geqslant 1)$ obtenue dans [2]. Il vient ainsi

$$
I \asymp t^{2} / u,
$$

ce qui fournit le résultat car $-I+R \asymp-t^{2} / u+O(1)$ lorsque $t>\sqrt{u}$. 
Notons cependant que l'on peut estimer $R$ plus précisément lorsque $t \gg \sqrt{u} \log u$ :

$$
\begin{aligned}
R & \ll \int_{1}^{u} \frac{\log (2 u / v) \mathrm{d} v}{\sqrt{v}+v \log (2 u / v-1)} \asymp \int_{1}^{2 u-1} \frac{u \log (w+1) \mathrm{d} w}{w \sqrt{w u}+u w \log w} \\
& \ll \int_{1}^{1+1 / \sqrt{u}} \sqrt{u}+\int_{1+1 / \sqrt{u}}^{2 u-1} \frac{\log (w+1) \mathrm{d} w}{w \log w} \ll \log u .
\end{aligned}
$$

Remarque. La démonstration précédente montre également que l'on peut remplacer $t / \sqrt{u}$ par $t / u$ dans le terme d'erreur de $(2 \cdot 5)$, dans tout domaine du type

$$
x \geqslant y \geqslant 2, \quad \vartheta(y) \geqslant(2+\varepsilon) \log x,
$$

puisque dans ce cas, $(2 \cdot 7)$ fournit $R \ll \int_{u-t}^{u} \mathrm{~d} v / v \ll \log (u /(u-t)) \ll t / u$ pour $t \leqslant u / 2$.

Le lemme suivant s'avère utile pour évaluer certaines sommes portant sur les nombres premiers.

Lemme 2.5. Nous avons, uniformément pour $x \geqslant y \geqslant 2$ et $2 \log x<\vartheta(y)$,

$$
\sum_{p \leqslant y} \frac{1}{p^{\alpha}}=\log _{2} y+\frac{u w}{w-1}\left(1+O\left(\frac{1}{\log y}+\frac{1}{\log 2 u}\right)\right),
$$

où $w:=\vartheta(y) / \log x$ et $\alpha=\alpha(x, y)$ est défini par $(2 \cdot 3)$.

Démonstration. Le lemme 3.6 de [3] fournit, pour $y \geqslant 2$,

$$
\sum_{p \leqslant y} \frac{1}{p^{\alpha}}=\log _{2} y+\left\{1+O\left(\frac{1-\alpha}{L(y)^{c_{11}}}\right)\right\} \int_{1}^{v_{\alpha}} t \xi^{\prime}(t) \mathrm{d} t+O(1),
$$

où nous avons posé

$$
v_{\alpha}:=\frac{y^{1-\alpha}-1}{(1-\alpha) \log y} .
$$

Il découle du lemme 2.7 de [2] que

$$
\log x=\frac{y^{1-\alpha}-1}{\left(1+y^{-\alpha}\right)(1-\alpha)}\left\{1+O\left(\frac{1}{\log y}\right)\right\}+O(1)
$$

et des formules (2.17) et (2.18) de [2] que

$$
1+y^{-\alpha}=\frac{w}{w-1}\left\{1+O\left(\frac{1}{\log y}\right)\right\} \quad(x \geqslant y \geqslant 2,2 \log x<\vartheta(y)) .
$$

Nous obtenons alors l'estimation

et la relation

$$
v_{\alpha}=\frac{u w}{w-1}\left\{1+O\left(\frac{1}{\log y}\right)\right\}
$$

$$
\xi^{\prime}(t)=\frac{1}{t}\left\{1+O\left(\frac{1}{\log 2 t}\right)\right\} \quad(t \geqslant 1)
$$

permet de conclure. 


\section{Comportement local de $\Psi_{f}(x, y)$}

\subsection{Martingale $y \mapsto \Psi_{f}(x, y)$ et inégalité maximale}

Soient $(\Omega, \mathcal{A}, \mathbb{P})$ un espace probabilisé, $\left\{X_{n}\right\}_{n \geqslant 0}$ une suite de variables aléatoires et $\mathcal{F}=\left\{\mathcal{F}_{n}\right\}_{n \geqslant 0}$ une filtration, i.e. une famille croissante de sous-tribus de $\mathcal{A}$.

Nous rappelons que $\left\{X_{n}\right\}_{n} \geqslant 0$ est dite une $\mathcal{F}$-martingale (resp. une $\mathcal{F}$-sousmartingale) si, pour tout entier $n \geqslant 0, X_{n}$ est $\mathcal{F}_{n}$-mesurable, $\mathbb{E}\left(\left|X_{n}\right|\right)<\infty$ et pour tout entier $n \geqslant 1$,

$$
\mathbb{E}\left(X_{n} \mid \mathcal{F}_{n-1}\right)=X_{n-1} \quad \text { ps } \quad\left(\text { resp. } \geqslant X_{n-1}\right) .
$$

Rappelons l'inégalité de Doob pour les sous-martingales positives (voir par exemple [13], §14.6, Theorem).

Lemme 3.1. Soit $\left\{X_{n}\right\}_{n \geqslant 0}$ une $\mathcal{F}$-sous-martingale positive. Pour tous $t>0$ et $n \geqslant 0$, nous avons

$$
\mathbb{P}\left(\max _{k \leqslant n} X_{k} \geqslant t\right) \leqslant \frac{\mathbb{E}\left(X_{n}\right)}{t} .
$$

Rappelons également l'assertion suivante (voir [13], §14.6, Lemma (b)).

Lemme 3.2. Si $\left\{X_{n}\right\}_{n \geqslant 0}$ est une $\mathcal{F}$-martingale, $\ell$ est un nombre entier $\geqslant 1$, et $\mathbb{E}\left|X_{n}^{\ell}\right|<\infty(n \geqslant 0)$, alors $\left\{X_{n}^{\ell}\right\}_{n \geqslant 0}$ est une $\mathcal{F}$-sous-martingale.

Considérant une fonction aléatoire au sens de Wintner $f$, nous désignons à présent par $\mathcal{F}_{y}:=\sigma\{f(p): p \leqslant y\}$ la tribu engendrée par les variables aléatoires $\{f(p)\}_{p \leqslant y}$, et par $\mathcal{F}$ la filtration $\left\{\mathcal{F}_{y}\right\}_{y \geqslant 2}$.

Proposition 3.3. Soit $f$ une fonction multiplicative aléatoire au sens de Wintner. Pour tout $x \geqslant 1$, la suite de variables aléatoires $\left\{\Psi_{f}(x, y)\right\}_{y \geqslant 2}$ est une $\mathcal{F}$-martingale.

Démonstration. Notons d'emblée que $\Psi_{f}(x, y)$ est $\mathcal{F}_{y}$-mesurable, pour $y \geqslant 2$. Par ailleurs, nous avons pour tout $y \geqslant 2$ premier,

$$
\begin{aligned}
\mathbb{E}\left(\Psi_{f}(x, y) \mid \mathcal{F}_{y-1}\right)= & \mathbb{E}\left(\Psi_{f}(x, y-1) \mid \mathcal{F}_{y-1}\right) \\
& \quad+\mathbb{E}\left(f(y) \Psi_{f}(x / y, y-1) \mid \mathcal{F}_{y-1}\right) \\
= & \Psi_{f}(x, y-1)+\Psi_{f}(x / y, y-1) \mathbb{E}(f(y)) \\
= & \Psi_{f}(x, y-1),
\end{aligned}
$$

car $\mathbb{E}(f(y))=0$.

Pour $y$ non premier, la relation précédente est bien encore vérifiée car $\Psi_{f}(x, y)=$ $\Psi_{f}(x, y-1)$.

Nous avons montré que $\left\{\Psi_{f}(x, y)\right\}_{y \geqslant 2}$ est une $\mathcal{F}$-martingale.

Le résultat suivant est un cas particulier du théorème 2.1 de [12]. 
Lemme 3.4. Soient $\left\{u_{n}\right\}_{n \geqslant 0}$ une suite de réels positifs, et $\left\{X_{n}\right\}_{n \geqslant 0}$ une suite de variables aléatoires. Supposons qu'il existe une constante $c_{12}>0$ telle que pour tout $n \geqslant 0$ et $t>0$, nous ayons

$$
\mathbb{P}\left(\sup _{k \leqslant n}\left|X_{k}\right| \geqslant t\right) \leqslant \frac{c_{12}}{t} \sum_{k \leqslant n} u_{k}
$$

Alors il existe une constante $c_{13}>0$, telle que pour toute suite croissante de réels positifs $\left\{v_{n}\right\}_{n \geqslant 0}$, nous ayons, pour tout $n \geqslant 0$ et $t>0$,

$$
\mathbb{P}\left(\sup _{k \leqslant n} \frac{\left|X_{k}\right|}{v_{k}} \geqslant t\right) \leqslant \frac{c_{13}}{t} \sum_{k \leqslant n} \frac{u_{k}}{v_{k}}
$$

Nous pouvons en déduire l'estimation suivante.

Lemme 3.5. Soit

$$
N_{j}:=\int_{1}^{X} \frac{\Psi_{f}\left(v, z_{j}\right)^{2}}{v^{2}} \mathrm{~d} v .
$$

Nous avons, pour tout $t>0$,

$$
\mathbb{P}\left(\sup _{j \leqslant J} \frac{N_{j}}{\log z_{j}}>t\right) \ll \frac{\log h}{t},
$$

avec $X \geqslant 2, z_{0} \geqslant 2, h:=\log X / \log z_{0}, \gamma>0, z_{j}:=z_{0}^{(1+\gamma)^{j}}(j \geqslant 1)$, $J:=\min \left\{j \geqslant 1: z_{j} \geqslant X\right\}$.

Démonstration. La relation $(2 \cdot 2)$ et l'estimation du théorème III.5.1 de [10]

$$
\Psi(x, y) \ll x \mathrm{e}^{-u / 2} \quad(x \geqslant y \geqslant 2)
$$

impliquent pour $v \geqslant 2$,

$$
\mathbb{E}\left(\Psi_{f}\left(v, z_{j}\right)^{2}\right)=\Psi^{*}\left(v, z_{j}\right) \leqslant \Psi\left(v, z_{j}\right) \ll v \mathrm{e}^{-(\log v) /\left(2 \log z_{j}\right)} .
$$

Par conséquent, pour tout $j \leqslant J$, nous obtenons

$$
\mathbb{E} N_{j}=\int_{1}^{X} \frac{\mathbb{E} \Psi_{f}\left(v, z_{j}\right)^{2}}{v^{2}} \mathrm{~d} v \ll \int_{1}^{X} \frac{\mathrm{e}^{-(\log v) /\left(2 \log z_{j}\right)}}{v} \mathrm{~d} v \ll \log z_{j} .
$$

Le Proposition 3.3 nous assure que $\left\{\Psi_{f}\left(v, z_{j}\right)\right\}_{j \leqslant J}$ est une $\left\{\mathcal{F}_{z_{j}}\right\}_{j \leqslant J}$-martingale et donc que la suite $\left\{N_{j} / \log z_{0}\right\}_{j \leqslant J}$ est une $\left\{\mathcal{F}_{z_{j}}\right\}_{j \leqslant J}$-sous-martingale. Nous avons 
ainsi, par l'inégalité de Doob pour les sous-martingales positives, pour tout $t>0$ et tout $j \leqslant J$,

$$
\mathbb{P}\left(\sup _{k \leqslant j} \frac{N_{k}}{\log z_{0}}>t\right) \leqslant \frac{\mathbb{E} N_{j}}{t \log z_{0}} \leqslant c_{14} \frac{(1+\gamma)^{j}}{t} \leqslant c_{14} \frac{\sum_{k \leqslant j} u_{k}}{t}
$$

où nous avons posé $u_{0}:=1$ et $u_{k}:=\gamma(1+\gamma)^{k}(k \geqslant 1)$. En effet, nous avons $\sum_{k \leqslant j} u_{k}=(1+\gamma)^{j+1}-\gamma \geqslant(1+\gamma)^{j}$ pour tout $j \leqslant J$.

Appliquons le Lemme 3.4 avec la suite croissante $v_{k}:=(1+\gamma)^{k}(k \geqslant 0)$, et il vient

$$
\mathbb{P}\left(\sup _{j \leqslant J} \frac{N_{j}}{\log z_{j}}>t\right)=\mathbb{P}\left(\sup _{j \leqslant J} \frac{N_{j}}{v_{j}\left(\log z_{0}\right)}>t\right) \leqslant c_{15} \frac{\sum_{j \leqslant J} u_{j} / v_{j}}{t} \ll \frac{1+\gamma J}{t},
$$

d'où le résultat, puisque nous avons $J \asymp(\log h) / \gamma$.

Remarque. La majoration triviale donnant

$$
\mathbb{P}\left(\sup _{j \leqslant J} \frac{N_{j}}{\log z_{j}}>t\right) \leqslant \sum_{j \leqslant J} \mathbb{P}\left(\frac{N_{j}}{\log z_{j}}>t\right) \ll \frac{J}{t} \asymp \frac{\log h}{\gamma t},
$$

le précédent lemme permet donc de gagner un facteur $1 / \gamma$.

\section{2. Étude des variations de $\Psi_{f}(x, y)$}

Nous ferons usage des notations additionnelles suivantes. Étant donné une suite strictement croissante d'entiers $\left\{x_{k}\right\}_{k \geqslant 1}$, nous posons, pour tout $j, k \geqslant 1$,

$$
y_{k, j}:=x_{k}^{1 / j}
$$

et désignons l'ensemble de tous les intervalles dyadiques inclus dans $\left.] x_{k-1}, x_{k}\right]$ par

$$
\left.\left.\mathcal{D}_{k}:=\left\{x_{k-1}+\right](s-1) 2^{m}, s 2^{m}\right]: s \geqslant 1, m \geqslant 0, s 2^{m} \leqslant x_{k}-x_{k-1}\right\} .
$$

En vue d'obtenir une majoration des quantités $\left|\Psi_{f}(x, y)-\Psi_{f}\left(x_{k}, y\right)\right|$, pour $x_{k-1}<x \leqslant x_{k}$ et $y \leqslant x_{k}$, nous établissons le lemme suivant, analogue du lemme 1 de [5]. La différence réside ici dans la prise en compte d'un paramètre de friabilité. Nous faisons ici pleinement usage de la martingale $y \mapsto \Psi_{f}(x, y)$, afin d'obtenir des majorations uniformes sur de larges plages du paramètre $y$. 
Lemme 3.6. Soient $f$ une fonction multiplicative aléatoire au sens de Wintner. Il existe des constantes $\left.c_{16} \in\right] 0,1\left[\right.$ et $c_{17}>0$ telles que, posant ${ }^{(3)}$

$$
x_{k}:=\left[\exp \left\{k^{c_{16}}\right\}\right], \quad J_{k}:=\max \left\{j \geqslant 1: 2 \log x_{k}<\vartheta\left(y_{k, j}\right)\right\} \quad(k \geqslant 1),
$$

nous ayons

$$
\max _{\substack{] a, b] \in \mathcal{D}_{k} \\ y \leqslant y_{k, j}}}\left|\Psi_{f}(b, y)-\Psi_{f}(a, y)\right| \ll \frac{\sqrt{\Psi^{*}\left(x_{k}, y_{k, j}\right)} \mathrm{e}^{c_{17} j}}{\log x_{k}} \quad\left(k \geqslant 1, j \leqslant J_{k}\right) \quad \text { ps. }
$$

Démonstration. Utilisant le Lemme 2.2 , nous avons pour tous entiers $b>a \geqslant 2$ et $y \geqslant 2$,

$$
\mathbb{E}\left\{\left(\Psi_{f}(b, y)-\Psi_{f}(a, y)\right)^{4}\right\} \leqslant\left\{\sum_{\substack{a<n \leqslant b \\ P^{+}(n) \leqslant y}} \mu(n)^{2} 3^{\omega(n)}\right\}^{2}=: E_{a, b}^{y} .
$$

Étant donnés $k \geqslant 1,1 \leqslant j \leqslant J_{k}$ et $R_{k, j} \geqslant 1$, un paramètre qui sera fixé ultérieurement, nous considérons l'événement

$$
A_{k, j}:=\left\{\max _{\substack{j a, b] \in \mathcal{D}_{k} \\ y \leqslant y_{k, j}}}\left|\Psi_{f}(b, y)-\Psi_{f}(a, y)\right| \geqslant R_{k, j}\right\} .
$$

Une application de l'inégalité de Doob à la sous-martingale positive (cf. Proposition 3.3 et Lemme 3.2) $\left\{\left(\Psi_{f}(b, y)-\Psi_{f}(a, y)\right)^{4}\right\}_{y \geqslant 2}$ fournit

$$
\mathbb{P}\left(\max _{y \leqslant y_{k, j}}\left|\Psi_{f}(b, y)-\Psi_{f}(a, y)\right| \geqslant R_{k, j}\right) \leqslant \frac{1}{R_{k, j}^{4}} E_{a, b}^{y_{k, j}} .
$$

Il vient alors, par sommation,

$$
\mathbb{P}\left(A_{k, j}\right) \leqslant \frac{1}{R_{k, j}^{4}} \sum_{] a, b] \in \mathcal{D}_{k}} E_{a, b}^{y_{k, j}}
$$

Utilisons à présent le fait que $E_{a, b}^{y}+E_{b, c}^{y} \leqslant E_{a, c}^{y}$ pour tous entiers $c>b>a \geqslant 2$ et $y \geqslant 2$, pour obtenir la majoration

$$
\begin{aligned}
\sum_{] a, b] \in \mathcal{D}_{k}} E_{a, b}^{y_{k, j}} & =\sum_{2^{m} \leqslant x_{k}-x_{k-1}} \sum_{s \leqslant\left(x_{k}-x_{k-1}\right) / 2^{m}} E_{x_{k-1}+(s-1) 2^{m}, x_{k-1}+s 2^{m}}^{y_{k, j}} \\
& \leqslant \sum_{2^{m} \leqslant x_{k}-x_{k-1}} E_{x_{k-1}, x_{k}}^{y_{k, j}} \ll\left(\log x_{k}\right) E_{x_{k-1}, x_{k}}^{y_{k, j} .}
\end{aligned}
$$

3. Nous désignons par $[x]$ la partie entière d'un nombre réel $x$. 
Ainsi

$$
\mathbb{P}\left(A_{k, j}\right) \ll \frac{\log x_{k}}{R_{k, j}^{4}} E_{x_{k-1}, x_{k}}^{y_{k, j}} .
$$

Nous avons, par l'inégalité de Hölder,

$$
\begin{aligned}
E_{x_{k-1}, x_{k}}^{y_{k, j}}=\left\{\sum_{\substack{x_{k-1}<n \leqslant x_{k} \\
P^{+}(n) \leqslant y_{k, j}}} \mu(n)^{2} 3^{\omega(n)}\right\}^{2} \\
\leqslant\left\{\Psi^{*}\left(x_{k}, y_{k, j}\right)-\Psi^{*}\left(x_{k-1}, y_{k, j}\right)\right\}^{4 / 3}\left\{\sum_{n \in S\left(x_{k}, y_{k, j}\right)} \mu(n)^{2} 3^{3 \omega(n)}\right\}^{2 / 3} .
\end{aligned}
$$

Nous avons, pour $x, y \geqslant 2$ et $\vartheta(y)>2 \log x$,

$$
\begin{aligned}
\sum_{n \in S(x, y)} \mu(n)^{2} 3^{3 \omega(n)} & \leqslant \sum_{d \in S(x, y)} \mu(d)^{2} 26^{\omega(d)} \Psi^{*}\left(\frac{x}{d}, y\right) \leqslant \Psi^{*}(x, y) \sum_{P^{+}(d) \leqslant y} \frac{\mu(d)^{2} 26^{\omega(d)}}{d^{\alpha}} \\
& \leqslant \Psi^{*}(x, y) \prod_{p \leqslant y}\left(1+\frac{26}{p^{\alpha}}\right) \leqslant \Psi^{*}(x, y)(\log y)^{26} \exp \left\{c_{17} u\right\}
\end{aligned}
$$

où nous avons fait appel à $(2 \cdot 5)$ et $(2 \cdot 8)$. En appliquant de plus le Lemme 2.3, valable sous réserve de l'existence d'une constante positive $K$ telle que

$$
\frac{x_{k}-x_{k-1}}{x_{k-1}} \geqslant \frac{1}{x_{k}^{K / j}}
$$

nous obtenons enfin, pour tout $j \leqslant J_{k}$,

$$
E_{x_{k-1}, x_{k}}^{y_{k, j}} \ll\left(\frac{x_{k}-x_{k-1}}{x_{k-1}}\right)^{4 / 3} \Psi^{*}\left(x_{k}, y_{k, j}\right)^{2}\left(\log x_{k}\right)^{52 / 3} \mathrm{e}^{c_{17} j},
$$

Considérons l'événement

$$
A_{k}:=\bigcup_{j \leqslant J_{k}} A_{k, j}
$$

et posons

$$
R_{k, j}:=\frac{\sqrt{\Psi^{*}\left(x_{k}, y_{k, j}\right)} \mathrm{e}^{c_{17} j}}{\log x_{k}} \quad\left(k \geqslant 1,1 \leqslant j \leqslant J_{k}\right) .
$$

Nous avons, compte tenu de (3.4),

$$
\begin{aligned}
\mathbb{P}\left(A_{k}\right) \leqslant \sum_{j \leqslant J_{k}} \mathbb{P}\left(A_{k, j}\right) & \ll\left(\frac{x_{k}-x_{k-1}}{x_{k-1}}\right)^{4 / 3}\left(\log x_{k}\right)^{67 / 3} \sum_{j \leqslant J_{k}} \mathrm{e}^{-3 c_{17} j} \\
& \ll\left(\frac{x_{k}-x_{k-1}}{x_{k-1}}\right)^{4 / 3}\left(\log x_{k}\right)^{67 / 3} .
\end{aligned}
$$


Soit $0<c_{16}<1$. Posons $x_{k}=\left[\exp \left\{k^{c_{16}}\right\}\right]$. L'estimation

$$
\frac{x_{k}-x_{k-1}}{x_{k-1}} \sim \frac{c_{16}}{k^{1-c_{16}}}
$$

assure tout d'abord que la condition (3.5) est bien vérifiée. En effet, le fait que $2 \log x_{k} \leqslant \vartheta\left(y_{k, j}\right)$ implique qu'il existe une constante $c_{18}>0$ telle que $x_{k}^{c_{18} / j} \geqslant \log x_{k}$. D'autre part, la relation (3.6) fournit

$$
\mathbb{P}\left(A_{k}\right) \ll \frac{1}{k^{5 / 4}},
$$

en choisissant par exemple $c_{16}=1 / 356$.

Le lemme de Borel-Cantelli permet alors d'obtenir

$$
\mathbb{P}\left(\limsup _{k \geqslant 1} A_{k}\right)=0
$$

puisque $\sum_{k \geqslant 1} \mathbb{P}\left(A_{k}\right)<\infty$. Ainsi, presque sûrement, il existe un entier $k_{0}$ tel que, pour tout $k \geqslant k_{0}$, et pour tout $j \leqslant J_{k}$,

$$
\max _{\substack{a, b] \in \mathcal{D}_{k} \\ y \leqslant y_{k, j}}}\left|\Psi_{f}(b, y)-\Psi_{f}(a, y)\right| \leqslant R_{k, j} .
$$

Corollaire 3.7. Sous les mêmes hypothèses, nous avons pour $k \geqslant 1$ et $j \leqslant J_{k}$,

$$
\max _{\substack{x_{k}-1 \leqslant x \leqslant x_{k} \\ y \leqslant y_{k, j}}}\left|\Psi_{f}(x, y)-\Psi_{f}\left(x_{k}, y\right)\right| \ll \sqrt{\Psi^{*}\left(x_{k}, y_{k, j}\right)} \mathrm{e}^{c_{17} j} \text { ps. }
$$

Démonstration. Soit $K \geqslant 0$. Tout entier positif $n \leqslant 2^{K+1}-1$ peut s'écrire

$$
n=\sum_{j \leqslant K} a_{j}(n) 2^{j} \quad\left(a_{j}(n) \in\{0,1\}, \forall j \in[0, K]\right) .
$$

Pour tout $k \geqslant 2$, le choix $K=\log \left(x_{k}-x_{k-1}+1\right) /(\log 2)-1$ est donc suffisant pour pouvoir écrire tout entier $n \leqslant x_{k}-x_{k-1}$ sous la forme (3.7). Cela implique, pour $x_{k-1}<x \leqslant x_{k}$, que tout intervalle $\left.] x_{k-1}, x\right]$ peut s'écrire comme la réunion disjointe d'au plus $O\left(\log x_{k}\right)$ intervalles dyadiques de $\mathcal{D}_{k}$, où $\mathcal{D}_{k}$ a été défini en $(3 \cdot 3)$.

Désignons alors par $\left.\left.\mathcal{D}(] x_{k-1}, x\right]\right)$ l'ensemble des intervalles dyadiques apparaissant dans la décomposition de $\left.] x_{k-1}, x\right]$ dont le nombre de termes est minimal.

Nous avons alors, pour tout $x_{k-1}<x \leqslant x_{k}$,

$$
\begin{aligned}
\left|\Psi_{f}(x, y)-\Psi_{f}\left(x_{k-1}, y\right)\right| & \leqslant \sum_{\substack{] a, b] \in \mathcal{D}(] x_{k-1}, x\right]\right)\\
}}\left|\Psi_{f}(b, y)-\Psi_{f}(a, y)\right| \\
& \ll\left(\log x_{k}\right) \max _{\substack{a, b] \in \mathcal{D}_{k} \\
y \leqslant y_{k, j}}}\left|\Psi_{f}(b, y)-\Psi_{f}(a, y)\right| .
\end{aligned}
$$

On conclut par le lemme précédent. 


\subsection{Moyenne de $\Psi_{f}(x, y)$ autour de points-tests}

Nous pouvons à présent démontrer le résultat suivant.

Lemme 3.8. Soit $f$ une fonction multiplicative aléatoire au sens de Wintner. Soit $\left\{x_{k}\right\}_{k \geqslant 1}$ définie comme au Lemme 3.6. Pour tout $\varepsilon>0$, il existe une constante $c_{19}>0$, telle que, uniformément pour

$$
\exp \left\{c_{19} \frac{\log x_{k}}{\log _{2} x_{k}}\right\} \leqslant y \leqslant x_{k}, \quad k \geqslant k_{0},
$$

nous ayons

$$
\frac{1}{x_{k}-x_{k-1}} \int_{x_{k-1}}^{x_{k}} \Psi_{f}(x, y) \mathrm{d} x \ll \sqrt{x_{k}}\left(\log _{2} x_{k}\right)^{2+\varepsilon} \quad \text { ps. }
$$

Démonstration. Il est nécessaire d'introduire tout d'abord un certain nombre de paramètres qui seront fixés ultérieurement. Soient donc $\gamma>0, X \geqslant 2$ et $z_{0} \geqslant 2$. Posons de plus

$$
k_{X}:=\left\{k \geqslant 1: \sqrt{X}<x_{k} \leqslant X\right\}, \quad h:=\frac{\log X}{\log z_{0}}
$$

en notant d'emblée que $\left|k_{X}\right| \leqslant(\log X)^{1 / c_{16}}$. Enfin, soit $\left\{z_{j}\right\}_{j} \geqslant 0$ une suite de nombres réels positifs définie de telle sorte que $z_{j}:=z_{j-1}^{1+\gamma}=z_{0}^{(1+\gamma)^{j}}(j \geqslant 1)$.

Nous pouvons, lorsque $2 \leqslant x \leqslant X$ et $y>z_{0}$, décomposer la somme $\Psi_{f}(x, y)$ selon la taille de $P^{+}(n)$ de la façon suivante

$$
\Psi_{f}(x, y)=\Psi_{f}\left(x, z_{0}\right)+\sum_{j \leqslant J_{0}(y)-1} \sum_{\substack{\left.\left.n \leqslant x \\ P^{+}(n) \in\right] z_{j}, z_{j+1}\right]}} f(n)+\sum_{\substack{\left.\left.n \leqslant x \\ P^{+}(n) \in\right] z_{J_{0}}, y\right]}} f(n)
$$

où l'on a posé $J_{0}=J_{0}(y):=\max \left\{j: z_{j} \leqslant y\right\}$ en observant que $J_{0}(y) \leqslant J:=$ $\min \left\{j: z_{j} \geqslant X\right\}$. Cela implique

$$
\begin{array}{r}
\Psi_{f}(x, y)=\Psi_{f}\left(x, z_{0}\right)+\sum_{j \leqslant J_{0}(y)-1} \sum_{1<r \leqslant x}^{z_{j}, z_{j+1}} f(r) \Psi_{f}\left(\frac{x}{r}, z_{j}\right) \\
+\sum_{1<r \leqslant x}^{z_{J_{0}}, y} f(r) \Psi_{f}\left(\frac{x}{r}, z_{J_{0}}\right) .
\end{array}
$$

Nous pouvons à présent écrire, pour la suite $\left\{x_{k}\right\}_{k \geqslant 1}$ définie au Lemme 3.6,

$$
\frac{1}{x_{k}-x_{k-1}}\left|\int_{x_{k-1}}^{x_{k}} \Psi_{f}(x, y)\right| \mathrm{d} x \leqslant V_{k}+\sum_{j \leqslant J} W_{k, j}^{*}
$$


avec

$$
\begin{aligned}
V_{k} & :=\frac{1}{x_{k}-x_{k-1}}\left|\int_{x_{k-1}}^{x_{k}} \Psi_{f}\left(x, z_{0}\right) \mathrm{d} x\right|, \\
W_{k, j}(z) & :=\frac{1}{x_{k}-x_{k-1}} \sum_{1<r \leqslant x_{k}}^{z_{j}, z} f(r) \int_{x_{k-1}}^{x_{k}} \Psi_{f}\left(\frac{x}{r}, z_{j}\right) \mathrm{d} x,
\end{aligned}
$$

et

$$
W_{k, j}^{*}:=\max _{z_{j}<z \leqslant z_{j+1}}\left|W_{k, j}(z)\right|, \quad Z_{k, j}:=W_{k, j}\left(z_{j+1}\right) .
$$

Soit $R$ un paramètre réel positif qui sera fixé ultérieurement (voir $(3 \cdot 17)$ ), et considérons les événements

$$
\begin{aligned}
A & :=\bigcup_{k \in k_{X}}\left\{\frac{1}{x_{k}-x_{k-1}} \max _{0}<y \leqslant x_{k}\left|\int_{x_{k-1}}^{x_{k}} \Psi_{f}(x, y) \mathrm{d} x\right| \geqslant 3 \sqrt{x_{k}} R\right\}, \\
B & :=\bigcup_{k \in k_{X}}\left\{V_{k} \geqslant \sqrt{x_{k}} R\right\}, \\
C_{j} & :=\bigcup_{k \in k_{X}}\left\{W_{k, j}^{*} \geqslant \sqrt{x_{k}} R / J\right\}, \quad C:=\bigcup_{j \leqslant J} C_{j} .
\end{aligned}
$$

La relation (3.9) implique directement

$$
A \subset B \cup C .
$$

Voyons comment majorer $\mathbb{P}(B)$. Il vient, par l'inégalité de Cauchy-Schwarz,

$$
\mathbb{E}\left(V_{k}^{2}\right) \leqslant \frac{1}{x_{k}-x_{k-1}} \int_{x_{k-1}}^{x_{k}} \mathbb{E} \Psi_{f}\left(x, z_{0}\right)^{2} \mathrm{~d} x \leqslant \Psi\left(x_{k}, z_{0}\right) .
$$

car, d'après la relation $(2 \cdot 2)$,

$$
\mathbb{E} \Psi_{f}\left(x, z_{0}\right)^{2}=\Psi^{*}\left(x_{k}, z_{0}\right) \leqslant \Psi\left(x_{k}, z_{0}\right) .
$$

L'inégalité de Markov et la majoration (3.2) pour $\Psi(x, y)$ impliquent

$$
\mathbb{P}\left(V_{k} \geqslant \sqrt{x_{k}} R\right) \leqslant \frac{\Psi^{*}\left(x_{k}, z_{0}\right)}{x_{k} R^{2}} \ll \frac{\mathrm{e}^{-\log x_{k} /\left(2 \log z_{0}\right)}}{R^{2}},
$$

et

$$
\mathbb{P}(B) \ll(\log X)^{1 / c_{16}} \frac{\mathrm{e}^{-h / 4}}{R^{2}} .
$$

Il nous reste donc à majorer $\mathbb{P}(C)$. Donnons-nous un entier pair $\ell \geqslant 2$ et introduisons la quantité

$$
D_{k, j}:=\frac{1}{x_{k}} \sum_{1<r \leqslant x_{k}}^{z_{j}, z_{j+1}} \mu(r)^{2}(\ell-1)^{\Omega(r)}\left\{\frac{1}{x_{k}-x_{k-1}} \int_{x_{k-1}}^{x_{k}} \Psi_{f}\left(\frac{x}{r}, z_{j}\right) \mathrm{d} x\right\}^{2} .
$$


Nous avons, par l'inégalité de Cauchy-Schwarz, pour tout $k \in k_{X}$,

$$
\begin{aligned}
D_{k, j} & \leqslant \frac{1}{x_{k}} \sum_{1<r \leqslant x_{k}}^{z_{j}, z_{j+1}} \mu(r)^{2}(\ell-1)^{\Omega(r)}\left\{\frac{1}{x_{k}-x_{k-1}} \int_{x_{k-1}}^{x_{k}} \Psi_{f}\left(\frac{x}{r}, z_{j}\right)^{2} \mathrm{~d} x\right\} \\
& \leqslant \sum_{1<r \leqslant x_{k}}^{z_{j}, z_{j+1}} \mu(r)^{2}(\ell-1)^{\Omega(r)}\left\{\frac{r / x_{k}}{x_{k}-x_{k-1}} \int_{x_{k-1} / r}^{x_{k} / r} \Psi_{f}\left(v, z_{j}\right)^{2} \mathrm{~d} v\right\} \\
& \leqslant \int_{1}^{x_{k} / z_{j}} \frac{\Psi_{f}\left(v, z_{j}\right)^{2}}{v}\left\{\frac{1}{x_{k}-x_{k-1}} \sum_{x_{k-1} / v<r \leqslant x_{k} / v} \mu(r)^{2}(\ell-1)^{\Omega(r)}\right\} \mathrm{d} v \\
& \leqslant \frac{c_{20} \ell \mathrm{e}^{2 \gamma \ell}}{\log z_{j}} \int_{1}^{x_{k} / z_{j}} \frac{\Psi_{f}\left(v, z_{j}\right)^{2}}{v^{2}} \mathrm{~d} v \leqslant \frac{c_{20} \ell \mathrm{e}^{2 \gamma \ell}}{\log z_{j}} N_{j} .
\end{aligned}
$$

où l'on utilise la notation $N_{j}$ définie en $(3 \cdot 1)$, et où la dernière somme en $r$ a été estimée par le Lemme 2.1, pourvu que l'on ait, pour $\delta>0$ convenablement fixé,

$$
z_{0}>\left(\frac{x_{k}}{x_{k}-x_{k-1}}\right)^{1+\delta}
$$

et

$$
\gamma \geqslant \frac{c_{21}}{L\left(z_{0}\right)^{c_{9}}} .
$$

Notons que la condition (3.12) est impliquée par l'inégalité

$$
h \leqslant c_{22} \frac{\log X}{\log _{2} X}
$$

où $h$ a été défini en $(3 \cdot 8)$. Les assertions $(3 \cdot 14)$ et $(3 \cdot 13)$ seront respectivement vérifiées en $(3 \cdot 16)$ et $(3 \cdot 18)$ lors du choix des paramètres $h$ et $\gamma$.

Soit à présent $R^{\prime}$ un paramètre réel positif fixé ultérieurement. Posons

$$
F_{j}:=\left\{N_{j} \leqslant R^{\prime} \cdot \log z_{j}\right\} .
$$

Nous sommes maintenant en mesure d'évaluer $\mathbb{P}(C)$. Nous avons

$$
C_{j}=\left(C_{j} \cap F_{j}\right) \cup\left(C_{j} \cap \overline{F_{j}}\right)
$$

d'où

$$
\begin{aligned}
C=\bigcup_{j \leqslant J} C_{j} & =\bigcup_{j \leqslant J}\left(C_{j} \cap F_{j}\right) \cup \bigcup_{j \leqslant J}\left(C_{j} \cap \overline{F_{j}}\right) \\
& \subset \bigcup_{j \leqslant J}\left(C_{j} \cap F_{j}\right) \cup \bigcup_{j \leqslant J} \overline{F_{j}}
\end{aligned}
$$


Grâce à l'inégalité de Doob appliquée à la sous-martingale $\left\{W_{k, j}(z)^{\ell}\right\}_{z>z_{j}}$ nous pouvons écrire, puisque $F_{j} \in \mathcal{F}_{z_{j}}$,

$$
\begin{aligned}
\mathbb{P}\left(C_{j} \cap F_{j}\right) & \leqslant \sum_{k \in k_{X}} \mathbb{P}\left(\left\{W_{k, j}^{*} \geqslant \sqrt{x_{k}} R / J\right\} \cap F_{j}\right) \\
& \leqslant \sum_{k \in k_{X}} \frac{\mathbb{E}\left(\mathbf{1}_{F_{j}} Z_{k, j}{ }^{\ell}\right)}{x_{k}^{\ell / 2}(R / J)^{\ell}} \leqslant \sum_{k \in k_{X}} \frac{\mathbb{E}\left(\mathbf{1}_{F_{j}} \mathbb{E}\left(Z_{k, j}{ }^{\ell} \mid \mathcal{F}_{z_{j}}\right)\right)}{x_{k}^{\ell / 2}(R / J)^{\ell}} .
\end{aligned}
$$

Une simple application du Lemme 2.2 fournit l'évaluation de l'espérance conditionnelle précédente sous la forme

$$
\mathbb{E}\left(Z_{k, j}{ }^{\ell} \mid \mathcal{F}_{z_{j}}\right) \leqslant\left(x_{k} \cdot D_{k, j}\right)^{\ell / 2} .
$$

Il en résulte alors

$$
\begin{aligned}
\mathbb{P}\left(C_{j} \cap F_{j}\right) & \leqslant \sum_{k \in k_{X}} \mathbb{E}\left(\mathbf{1}_{F_{j}} \cdot \frac{D_{k, j}^{\ell / 2}}{(R / J)^{\ell}}\right) \\
& \leqslant(\log X)^{1 / c_{16}} \mathrm{e}^{\gamma \ell^{2}}\left(\frac{c_{20} \cdot \ell \cdot R^{\prime} \cdot J^{2}}{R^{2}}\right)^{\ell / 2} .
\end{aligned}
$$

D'autre part, le Lemme 3.5 fournit

$$
\mathbb{P}\left(\bigcup_{j \leqslant J} \overline{F_{j}}\right)=\mathbb{P}\left(\max _{j \leqslant J} \frac{N_{j}}{\log z_{j}}>R^{\prime}\right) \ll \frac{\log h}{R^{\prime}} .
$$

Il découle de $(3 \cdot 10),(3 \cdot 11),(3 \cdot 15)$ et du fait que $J \asymp \log h / \gamma$, que

$$
\begin{aligned}
\mathbb{P}(A) & \leqslant \mathbb{P}(B)+\mathbb{P}(C) \\
& \ll(\log X)^{1 / c_{16}}\left\{J^{\ell+1}\left(\frac{c_{20} \cdot \ell \cdot R^{\prime}}{R^{2}}\right)^{\ell / 2} \mathrm{e}^{\gamma \ell^{2}}+\frac{\mathrm{e}^{-h / 4}}{R^{2}}\right\}+\frac{\log h}{R^{\prime}} \\
& \leqslant(\log X)^{1 / c_{16}}\left\{\frac{(\log h)^{\ell+1}}{\gamma^{\ell+1}}\left(\frac{c_{20} \cdot \ell \cdot R^{\prime}}{R^{2}}\right)^{\ell / 2} \mathrm{e}^{\gamma \ell^{2}}+\frac{\mathrm{e}^{-h / 4}}{R^{2}}\right\}+\frac{\log h}{R^{\prime}} .
\end{aligned}
$$

Prenons $\gamma:=1 / \ell$. Il vient

$$
\mathbb{P}(A) \leqslant(\log X)^{c_{23}}\left\{\left(\frac{\sqrt{R^{\prime} \log h}}{R}\right)^{\ell}\left(c_{24} \ell\right)^{3 \ell / 2+1}+\frac{\mathrm{e}^{-h / 4}}{R^{2}}\right\}+\frac{\log h}{R^{\prime}} .
$$

Le choix

$$
\ell=2\left[\frac{1}{2}\left(\frac{R}{\left(\sqrt{R^{\prime}} \log h\right)}\right)^{2 / 3}\right]
$$


conduit à l'estimation

$$
\mathbb{P}(A) \ll(\log X)^{c_{23}}\left\{\exp \left\{-\frac{c_{25} R^{2 / 3}}{R^{1 / 3}(\log h)^{2 / 3}}\right\}+\frac{\mathrm{e}^{-h / 4}}{R^{2}}\right\}+\frac{\log h}{R^{\prime}} .
$$

Remarquons qu'en fixant

$$
h=c_{26} \log _{2} X,
$$

nous avons

$$
\mathbb{P}(A) \ll(\log X)^{c_{23}} \exp \left\{-\frac{c_{25} R^{2 / 3}}{\left(R^{\prime 1 / 3}\left(\log _{3} X\right)^{2 / 3}\right)}\right\}+\frac{1}{R^{2}}+\frac{\log _{3} X}{R^{\prime}},
$$

et que la condition $(3 \cdot 14)$ est bien remplie, comme annoncé précédemment. Le choix $R^{\prime}=c_{27}\left(\log _{2} X\right)^{1+\varepsilon}$ fournit enfin

$$
\mathbb{P}(A) \ll(\log X)^{c_{23}} \exp \left\{-\frac{c_{25} R^{2 / 3}}{\left(\log _{2} X\right)^{1 / 3+\varepsilon}}\right\}+\frac{1}{R^{2}}+\frac{1}{\left(\log _{2} X\right)^{1+\varepsilon / 2}} .
$$

Nous pouvons ainsi fixer

$$
R=\left(\log _{2} X\right)^{2+\varepsilon},
$$

en notant que dans ce cas, la condition $(3 \cdot 13)$ est bien vérifiée puisque

$$
\gamma \gg\left(\frac{\sqrt{R^{\prime}} \log h}{R}\right)^{2 / 3} \gg \frac{1}{\sqrt{\log X}} \gg \frac{1}{L\left(z_{0}\right)^{c_{9}}} .
$$

Posant $X=X_{s}:=\exp \left\{2^{s}\right\}$, nous avons clairement

$$
\mathbb{P}\left(A_{X_{s}}\right) \leqslant \frac{1}{s^{1+\varepsilon}} .
$$

Le lemme de Borel-Cantelli permet alors d'établir, puisque la série $\sum_{s \geqslant 1} \mathbb{P}\left(A_{X_{s}}\right)$ est convergente, que

$$
\mathbb{P}\left(\limsup _{s \geqslant 1} A_{X_{s}}\right)=0 .
$$

Il existe ainsi, presque sûrement, un entier $k_{1}$ tel que pour $k \geqslant k_{1}$,

$$
\max _{z_{0}<y \leqslant x_{k}} \frac{1}{x_{k}-x_{k-1}} \int_{x_{k-1}}^{x_{k}} \Psi_{f}(x, y) \mathrm{d} x \leqslant 3 \sqrt{x_{k}} R .
$$


JOSEPH BASQUIN

\section{Preuve du Théorème 1.2}

Pour $x$ fixé, nous définissons $k_{1}$ comme l'unique entier tel que

$$
x_{k_{1}-1}<x \leqslant x_{k_{1}},
$$

Nous scindons la démonstration du Théorème 1.2 en plusieurs cas, selon la taille relative de $y$ par rapport à $x$.

Premier cas.

Supposons $x \geqslant y \geqslant \exp \left\{c_{19} \log x / \log _{2} x\right\}$. Nous avons, dans ce domaine,

$$
\begin{gathered}
\left|\Psi_{f}(x, y)\right| \leqslant \min _{x_{k_{1}-1}<x^{\prime} \leqslant x_{k_{1}}}\left|\Psi_{f}\left(x^{\prime}, y\right)\right|+\max _{x_{k_{1}-1}<x^{\prime} \leqslant x_{k_{1}}}\left|\Psi_{f}\left(x_{k_{1}}, y\right)-\Psi_{f}\left(x^{\prime}, y\right)\right| \\
\leqslant \frac{1}{x_{k_{1}}-x_{k_{1}-1}} \int_{x_{k_{1}-1}}^{x_{k_{1}}} \Psi_{f}\left(x^{\prime}, y\right) \mathrm{d} x^{\prime} \\
\quad+\max _{x_{k_{1}-1}<x^{\prime} \leqslant x_{k_{1}}}\left|\Psi_{f}\left(x_{k_{1}}, y\right)-\Psi_{f}\left(x^{\prime}, y\right)\right| .
\end{gathered}
$$

Il vient alors

$$
\Psi_{f}(x, y) \ll \sqrt{x_{k_{1}}}\left(\log _{2} x_{k_{1}}\right)^{2+\varepsilon}+x_{k} \ll \sqrt{x}\left(\log _{2} x\right)^{2+\varepsilon},
$$

en appliquant respectivement le Corollaire 3.7 et le Lemme 3.8 .

Deuxième cas.

Nous supposons ici que l'on a $\vartheta(y)>2 \log x$. Posons à présent

$$
j_{k}:=\max \left\{1,\left[\frac{\log x_{k}}{\log y}\right]\right\}, \quad y_{k}:=x_{k}^{1 / j_{k}} \quad\left(k \leqslant k_{1}\right) .
$$

Le Corollaire 3.7, fournit directement

$$
\left|\Psi_{f}\left(x_{k}, y\right)-\Psi_{f}\left(x_{k-1}, y\right)\right| \leqslant \sqrt{\Psi^{*}\left(x_{k}, y_{k}\right)} \mathrm{e}^{c_{17} j_{k}} \quad\left(k \leqslant k_{1}\right)
$$

et

$$
\left|\Psi_{f}\left(x_{k_{1}}, y\right)-\Psi_{f}(x, y)\right| \leqslant \sqrt{\Psi^{*}\left(x_{k_{1}}, y_{k_{1}}\right)} \mathrm{e}^{c_{17} j_{k_{1}}} .
$$

Notons que, pour tout $k \leqslant k_{1}$, nous avons

$$
\begin{aligned}
j_{k} & \leqslant \max \left\{1, \frac{\log x_{k}}{\log y}\right\} \leqslant \frac{\log x_{k_{1}}}{\log y} \leqslant \frac{\log x_{k_{1}-1}}{\log y}+\frac{\log \left(x_{k_{1}} / x_{k_{1}-1}\right)}{\log y} \\
& \leqslant u+O\left(\frac{1}{\log y}\right),
\end{aligned}
$$


en vertu de la relation $(4 \cdot 1)$. Supposant provisoirement l'estimation

$$
M_{1}:=\sup _{k \leqslant k_{1}} \frac{\Psi^{*}\left(x_{k}, y_{k}\right)}{\Psi^{*}(x, y)}=\exp \left\{O\left(\frac{u}{L_{\varepsilon}(y)}+\log (u+1)\right)\right\}
$$

acquise, nous pouvons en déduire la majoration

$$
\begin{aligned}
\left|\Psi_{f}(x, y)\right| & \leqslant \sum_{k \leqslant k_{1}}\left|\Psi_{f}\left(x_{k}, y\right)-\Psi_{f}\left(x_{k-1}, y\right)\right|+\left|\Psi_{f}(x, y)-\Psi_{f}\left(x_{k_{1}}, y\right)\right| \\
& \ll k_{1} \sqrt{M_{1}} \sqrt{\Psi^{*}(x, y)} \mathrm{e}^{c_{17} u} \ll \sqrt{\Psi^{*}(x, y)} \mathrm{e}^{c_{28} u}(\log x)^{c_{29}},
\end{aligned}
$$

où l'on a utilisé le fait que $k_{1} \ll(\log x)^{1 / c_{16}}$.

Établissons à présent la relation $(4 \cdot 3)$. Lorsque $x$ est suffisamment grand et $(\log x)^{3}<y \leqslant x$, nous avons $\beta(x, y) \geqslant 3 / 5$. En appliquant le corollaire 2.6 de [3] et le théorème III.5.21 de [10], il vient

$$
\begin{aligned}
M_{1} & \asymp \sup _{k \leqslant k_{1}} \frac{\Psi\left(x_{k}, y_{k}\right)}{\Psi(x, y)} \\
& \leqslant \sup _{k \leqslant k_{1}} \frac{x_{k}}{x} \frac{\varrho\left(\log x_{k} / \log y-1\right)}{\varrho(u)} \exp \left\{O\left(\frac{\log (u+1)}{\log y}+\frac{u}{L_{\varepsilon}(y)}+\frac{1}{u}\right)\right\} .
\end{aligned}
$$

Faisant usage de la croissance de la fonction $x \mapsto x \varrho(\log x / \log y-1)$ pour $x$ suffisamment grand et $y \leqslant x \leqslant \exp \left\{y^{1 / 3}\right\}$, nous obtenons

$$
\begin{aligned}
M_{1} & \leqslant \frac{x_{k_{1}}}{x} \frac{\varrho\left(\log x_{k_{1}} / \log y-1\right)}{\varrho(u)} \exp \left\{O\left(\frac{\log (u+1)}{\log y}+\frac{u}{L_{\varepsilon}(y)}+\frac{1}{u}\right)\right\} \\
& =\exp \left\{O\left(\frac{u}{L_{\varepsilon}(y)}+\log (u+1)\right)\right\} .
\end{aligned}
$$

Plaçons-nous à présent dans le domaine

$$
2 \log x<\vartheta(y) \leqslant(\log x)^{3} .
$$

Puisque $j_{k} \geqslant \log x_{k} / \log y-1$, nous avons $y \leqslant y_{k} \leqslant y+y^{3 / 4}$ si l'on suppose de plus que

$$
x_{k} \geqslant \exp \left\{y^{1 / 4}(\log y)^{2}\right\}
$$

En remarquant que nous avons trivialement

$$
\Psi^{*}\left(x_{k}, y_{k}\right) \leqslant x_{k} \leqslant \exp \left\{O\left(\frac{u}{L_{\varepsilon}(y)}\right)\right\}
$$


pour tous les entiers $k$ ne vérifiant pas $(4 \cdot 5)$, et que $x_{k} \leqslant \mathrm{e} x\left(k \leqslant k_{1}\right)$, il vient

$$
\begin{gathered}
M_{1} \ll \frac{\Psi^{*}\left(x, y+y^{3 / 4}\right)}{\Psi^{*}(x, y)}=1+\sum_{\substack{d>1 \\
P^{-}(d)>y, P^{+}(d) \leqslant y+y^{3 / 4}}} \mu(d)^{2} \frac{\Psi^{*}(x / d, y)}{\Psi^{*}(x, y)} \\
\ll 1+\sum_{\substack{d>1 \\
P^{-}(d)>y, P^{+}(d) \leqslant y+y^{3 / 4}}} \frac{\mu(d)^{2}}{d^{\alpha}} \ll \exp \left\{\sum_{y<p \leqslant y+y^{3 / 4}} \frac{1}{p^{\alpha}}\right\},
\end{gathered}
$$

où $\alpha$ a été défini en $(2 \cdot 3)$. Posant

$$
w(t)=w(t ; x, y):=\frac{t^{1-\alpha}-1}{(1-\alpha) \log t} \quad(t \geqslant 2),
$$

le lemme 3.6 de [3] fournit, pour $y \geqslant 2$,

$$
\begin{aligned}
\sum_{y<p \leqslant y+y^{3 / 4}} \frac{1}{p^{\alpha}} & =\log \left(\frac{\log \left(y+y^{3 / 4}\right)}{\log y}\right)+\int_{w(y)}^{w\left(y+y^{3 / 4}\right)} t \xi^{\prime}(t) d t+O\left(\frac{y^{1-\alpha}}{L(y)^{c_{30}}}\right) \\
& \ll w\left(y+y^{3 / 4}\right)-w(y)+\frac{y^{1-\alpha}}{L(y)^{c_{30}}} \ll \frac{y^{3 / 4}}{y^{\alpha} \log y}+\frac{y^{1-\alpha}}{L(y)^{c_{30}}} \\
& \ll \frac{u}{L_{\varepsilon}(y)},
\end{aligned}
$$

car on a $y^{1-\alpha} \asymp \log x$ dans le domaine considéré. Cela achève la démonstration de l'estimation (4.3) et donc de $(4 \cdot 4)$.

Un calcul simple permet enfin d'observer que l'estimation (4.4) est meilleure que celle résultant de $(4 \cdot 2)$ si

$$
x \geqslant 16, \quad y \leqslant \exp \left\{c_{31} \frac{\log x \log _{3} x}{\log _{2} x}\right\} .
$$

où $c_{31}$ est une constante positive convenable.

Troisième cas.

Il s'agit à présent d'évaluer le domaine dans lequel la majoration triviale

$$
\left|\Psi_{f}(x, y)\right| \leqslant \Psi^{*}(x, y)
$$

est meilleure que l'estimation (4.4). Nous supposons ici que $\vartheta(y)>2 \log x$.

D'une part, pour tout $\varepsilon>0$, il existe $\delta>0$ tel que l'on ait

$$
v:=\log (\vartheta(y) / \log x-1) \leqslant \varepsilon \log y,
$$


lorsque $y \leqslant(\log x)^{1+\delta}$. Aussi, sous cette dernière hypothèse, le corollaire 2.3 de [2] fournit la minoration

$$
\Psi^{*}(x, y) \geqslant \exp \left\{(1-\varepsilon) \pi(y) v /\left(\mathrm{e}^{v}+1\right)\right\} \geqslant \exp \left\{\left(1-\varepsilon^{\prime}\right) v u\right\},
$$

d'où

$$
\Psi^{*}(x, y) \gg \sqrt{\Psi^{*}(x, y)} \mathrm{e}^{c_{4} u}(\log x)^{c_{5}}
$$

lorsque $v$ est suffisamment grand.

D'autre part, lorsque $y \geqslant(\log x)^{1+\delta}$, il découle de l'estimation de $\Psi(x, y)$ par Hildebrand et Tenenbaum (théorème III.5.21 de [10]) que

$$
\sqrt{\Psi^{*}(x, y)} \asymp \sqrt{\Psi(x, y)} \gg \mathrm{e}^{c_{4} u}(\log x)^{c_{5}}
$$

et donc que l'estimation (4.4) est toujours de qualité supérieure à la majoration triviale (4.6) lorsque $y \geqslant(\log x)^{1+\delta}$ et $x$ est suffisamment grand.

$\mathrm{Au}$ vu des observations précédentes, la majoration fournie par (4.4) est meilleure que la majoration triviale (4.6) lorsque $\vartheta(y) \geqslant c_{32} \log x$, où $c_{32}$ est une constante positive.

\section{Preuve du Corollaire 1.3}

Déterminons dans quel domaine les majorations apportées par le Théorème 1.2 fournissent une estimation du type $\Psi_{f}(x, y) \ll_{\varepsilon} \Psi^{*}(x, y)^{1 / 2+\varepsilon}$ pour tout $\varepsilon>0$. Tout d'abord, il apparait clairement (en utilisant par exemple la formule asymptotique issue du corollaire III.5.19 de [10]) que l'on a

$$
\sqrt{x}\left(\log _{2} x\right)^{2+\varepsilon^{\prime}} \ll \Psi(x, y)^{1 / 2+\varepsilon} \asymp \Psi^{*}(x, y)^{1 / 2+\varepsilon}
$$

lorsque $x \geqslant y \geqslant x^{c_{3}\left(\log _{3} x\right) / \log _{2} x}$ et $x$ suffisamment grand.

Par ailleurs, pour $(\log x)^{1+\delta} \leqslant y \leqslant x^{c_{3}\left(\log _{3} x\right) / \log _{2} x}$ et $x$ suffisamment grand, le théorème III.5.21 de [10] nous assure que

$$
\Psi^{*}(x, y) \mathrm{e}^{c_{4} u}(\log x)^{c_{5}} \ll \Psi^{*}(x, y)^{1 / 2+\varepsilon}
$$

puisque l'on a alors

$$
\Psi^{*}(x, y)^{\varepsilon} \asymp \Psi(x, y)^{\varepsilon} \gg \mathrm{e}^{c_{4} u}(\log x)^{c_{5}} .
$$

Enfin, la minoration (4.8), valable dans le domaine $y \leqslant(\log x)^{1+\delta}, \vartheta(y)>2 \log x$ nous assure que

$$
\Psi^{*}(x, y)^{\varepsilon} \gg \mathrm{e}^{c_{4} u}(\log x)^{c_{5}}
$$


lorsque $\varepsilon v$ est suffisamment grand (rappelons que $v$ est défini en (4·7)), c'est-à-dire lorsque $y \geqslant C_{\varepsilon} \log x$. Ceci implique directement la majoration

$$
\Psi^{*}(x, y) \mathrm{e}^{c_{4} u}(\log x)^{c_{5}} \ll \Psi^{*}(x, y)^{1 / 2+\varepsilon} .
$$

Remerciements. L'auteur remercie Gérald Tenenbaum pour l'aide qu'il lui a apportée au cours de l'élaboration de ce travail, et tient également à exprimer sa gratitude à l'arbitre, pour ses remarques éclairantes.

\section{Bibliographie}

[1] A. Bonami, Étude des coefficients de Fourier des fonctions de $L^{p}(G)$, Ann. Inst. Fourier 20 (1970), 335-402.

[2] R. de la Bretèche, G. Tenenbaum, Sur les lois locales de la répartition du k-ième diviseur d'un entier, Proc. London Math. Soc. (3) 84 (2002).

[3] R. de la Bretèche, G. Tenenbaum, Propriétés statistiques des entiers friables, Ramanujan J. 9 (2005), 139-202.

[4] P. Erdős, Some unsolved problems, Magyar Tud. Akad. Kutató Int. Közl. 6 (1961), $221-254$.

[5] G. Halász, On random multiplicative functions, Hubert Delange colloquium (Orsay, 1982), Publ. Math. Orsay 83 (4) (1983), 74-96.

[6] A. J. Harper, On the limit distributions of some sums of a random multiplicative function, à paraître.

[7] A. Hildebrand, G. Tenenbaum, On integers free of large prime factors, Trans. Amer. Math. Soc. 296 (1986), 265-290.

[8] A. Hildebrand, G. Tenenbaum, Integers without large prime factors. J. Théor. Nombres Bordeaux 5 (1993), no. 2, 411-484.

[9] Y.-K. Lau, G. Tenenbaum, J. Wu, Mean values of random multiplicative functions, prépublication, sept. 2010 ; version finale : Proc. Amer. Math. Soc., à paraître.

[10] G. Tenenbaum, Introduction à la théorie analytique et probabiliste des nombres, Troisième Edition, Belin, 2008.

[11] G. Tenenbaum, J. Wu, Moyennes de certaines fonctions multiplicatives sur les entiers friables, J. Reine Angew. Math. 564 (2003), 119-166.

[12] T. Tómács, Z. Líbor, A Hájek-Renyi type inequality and its applications, Ann. Math. et Inform. 33 (2006), 141-149.

[13] D. Williams, Probability with martingales, Cambridge University Press, 1991.

[14] A. Wintner, Random factorizations and Riemann's hypothesis, Duke Math. J. 11 (1944), 267-275.

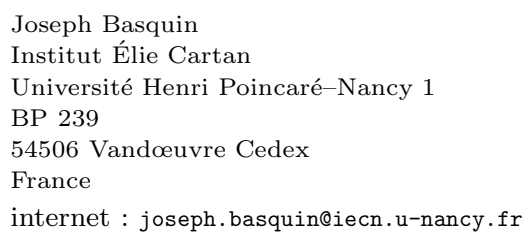

